\section{Staff experiences of the REFOCUS intervention to support recovery in mental health: A qualitative study nested within a cluster randomized controlled trial}

\author{
E Clarke $^{1 \#, ~ M ~ L e a m y ~}{ }^{2 * \#}$, V Bird ${ }^{1}$, M Janosik ${ }^{1}$, H Jordan ${ }^{1}$, C Le \\ Boutillier $^{1}$, R Macpherson², G Riley², K Sanger ${ }^{2}$, J Williams ${ }^{1}$ \\ and M Slade ${ }^{1}$ \\ ${ }^{1}$ King's College London, Health Service and Population Research Department, Denmark Hill, \\ Institute of Psychiatry, London, SE5 8AF, UK \\ ${ }^{2}$ King's College London, Mental Health Nursing Department, Florence Nightingale Faculty of \\ Nursing, Midwifery and Palliative care, Waterloo campus, SE1 8WA, UK \\ \#Joint first authors \\ ${ }^{2}$ gether NHS Foundation Trust Headquarters, Rikenel, Montpellier, Gloucester, GL1 1LY
}

\section{Abstract}

Background: The REFOCUS intervention was a whole team, complex intervention, designed to increase the recovery support offered by community based, mental health staff. The intervention consisted of two components: Recovery promoting relationships, which focused on how staff work with service users, and Recovery working practices, which focused on what activities and tasks staff and service users could do together.

Aim: We aimed to investigate the experiences of community mental health workers using the REFOCUS intervention to support personal recovery.

Method: In the context of the REFOCUS Trial (ISRCTN02507940), 28 semi-structured individual interviews and 4 staff focus groups, with 24 participants were conducted and thematically analyzed.

Results: Staff valued coaching training and used coaching skills to have tough as well as empowering, motivational conversations with service users. They were positive about the resources within the 'working practices' intervention component. The whole team training and reflection sessions helped create team cultures, structures and processes which were conducive to supporting recovery practice.

Conclusion: We recommend the wider use of coaching skills, strengths-based assessments, and approaches to support clinicians to broaden their understanding of service users' values, treatment preferences and to support striving towards personally-meaningful goals. Staff who used these working practices changed their beliefs about what their service users were capable of, and became more hopeful practitioners. A team-based approach to support recovery creates a learning environment in which staff can support and challenge one another, making sustained practice change more likely.

\section{More Information}

*Address for Correspondence: M Leamy, King's College London, Mental Health Nursing Department, Florence Nightingale Faculty of Nursing, Midwifery and Palliative care, Waterloo campus, SE1 8WA, UK, Email: mary.leamy@kcl.ac.uk

\section{Submitted: 06 March 2020}

Approved: 24 April 2020

Published: 27 April 2020

How to cite this article: Clarke $E$, Leamy M, Bird V, Janosik M, Jordan H, et al. Staff experiences of the REFOCUS intervention to support recovery in mental health: A qualitative study nested within a cluster randomized controlled trial. Arch Psychiatr Ment Health. 2020; 4: 024-032.

DOI: 10.29328/journal.apmh.1001014

Copyright: @ 2020 Clarke E, et al. This is an open access article distributed under the Creative Commons Attribution License, which permits unrestricted use, distribution, and reproduction in any medium, provided the original work is properly cited.

Keywords: Recovery; Complex interventions; Process evaluation; Psychosis; Cluster randomized controlled trial; Staff experiences; REFOCUS

\section{Check for updates}

OPEn ACCESS

\section{Introduction}

Over the last couple of decades, mental health systems in England, Australia, New Zealand, United States, Canada and other English-speaking countries have adopted a recoveryoriented framework within mental health policy and practice
[1-3]. Supporting personal recovery involves an emphasis on personalized care so service users can live a satisfying, hopeful and contributing life [4]. The literature providing guidance on how to implement recovery practice has been growing, though evidence suggests it remains problematic $[5,6]$. In a recent grounded theory of staff and manager perceptions 
of the barriers and enablers to supporting recovery, a core category of 'Competing Priorities' emerged, where staff struggle to make sense of recovery-oriented practice in the face of conflicting demands, informed by different priorities of different health system levels [7]. System transformation of mental health services remains a key challenge [8] and needs to be supplemented by other initiatives to ensure recovery outcomes are measured and organizational commitment is demonstrated to staff consistently [9].

Staff and service provider attitudes are a key determinant in the provision of recovery-oriented care [10]. Fundamentally, successful and sustained implementation of recovery practice depends on staff believing in the ability of service users to live better, more fulfilling lives, despite the continuing limitations resulting from their illness and in the ability of service users to live beyond the illness [11]. A pro-recovery, complex intervention, designed to change staff attitudes, knowledge, skills and behavior was evaluated in a cluster randomized controlled trial (RCT), as part of the REFOCUS research programme [12]. The intervention consisted of two components: i) Recovery working practices, which comprised of tasks and activities which support recovery, and ii) Recovery-promoting relationships, which focused on how staff should relate to service users [13] (see table 1 for summary of REFOCUS components) . Within the Recovery working practices component, staff were asked to have three specific conversations and/or carry out activities to support recovery. These were i) to understand values and treatment preferences, ii) to assess and amplify strengths, and iii) to support goal-striving. The Recovery-promoting relationships component was supported by providing teams with training and reflection opportunities to help them develop a shared team knowledge and understanding of recovery and reflect upon their own personal and team values. The intervention and evaluation was based on best practice in recovery support [14], and systematic reviews of personal recovery [15], (which have been validated with current service users [16] and cross-culturally [17]), strengths [18], recovery measures [19] and recovery support measures [20]. The staff intervention manual [13] contained the REFOCUS model, based on the theory of planned behavior, showing the pathway from staff receiving the Intervention, through Practice change, targeting team and individual underlying recovery values, level of knowledge about personal recovery, skills in coaching and the three working practices, to Behavioral intent and Actual behaviour, leading to an improved Service user experience, in terms of content of care (more experience of coaching, greater focus on strengths, values and goal-striving) and process (more support for personal recovery) and Outcomes for service users (proximal outcomes such as hopefulness, empowerment, quality of life and well-being and distal outcome of improved personal recovery).

In line with best practice in trial methodology [21], we undertook a process evaluation of participant experiences of delivering and receiving the intervention. The study reported here specifically aimed to investigate the experiences of community mental health workers using the complex intervention to support personal recovery and views on individual intervention components. Staff, manager and trainer perceptions on the implementation of the intervention, service user perceptions on receiving the intervention, a description of the development of the REFOCUS intervention, and quantitative findings from the trial are reported elsewhere $[22,33,35]$.

\section{Methods}

\section{Participants}

Participants were invited to participate in an individual, semi-structured interview if they self-reported the use of either the working practices or coaching skills. Interviewees were purposively sampled to maximise variation within the sample of profession, gender, trial site (South London and Maudsley NHS trust and ${ }^{2}$ gether NHS trust) and wave (entry to trial was staggered).

A total of 49 staff (28 interviews; 24 in focus groups; with 3 staff in both) participated. They came from Assertive outreach, Support and Recovery, Forensic and Psychosis community based mental health teams. Their socio-demographic characteristics are shown in table 3.

\section{Intervention}

Participants received two types of training, Personal Recovery training and coaching for Recovery training, to

\begin{tabular}{|c|}
\hline Table 1: Summary of REFOCUS intervention components. \\
\hline Component 1: Recovery-promoting relationships \\
\hline Developing a shared team understanding of personal recovery \\
Exploring individual and team values \\
Skills training in coaching \\
\hline Teams carrying out partnership project with service users \\
\hline Raising the expectations held by service users that their values, strengths and \\
goals will be prioritised \\
\hline Component 2: Working practices \\
\hline Values and treatment preferences \\
\hline Strengths \\
\hline Personally- valued goals \\
\hline
\end{tabular}

\begin{tabular}{|c|c|c|c|}
\hline Implementation strategy & To whom & Length of time & Month \\
\hline $\begin{array}{c}\text { Information sessions for staff } \\
\text { and service users }\end{array}$ & $\begin{array}{l}\text { Provided to } \\
\text { team }\end{array}$ & 1 hour & Month 1 \\
\hline Personal recovery training & $\begin{array}{l}\text { Provided to } \\
\text { team }\end{array}$ & $3 x$ half days & Month $1,2 \& 5$ \\
\hline $\begin{array}{l}\text { Coaching conversations for } \\
\text { Recovery training }\end{array}$ & $\begin{array}{l}\text { Provided to } \\
\text { team }\end{array}$ & $\begin{array}{l}1 \text { full and } 2 \text { half } \\
\text { days }\end{array}$ & Month $3,4,5$ \\
\hline Team reflection sessions & $\begin{array}{l}3 \text { externally } \\
\text { facilitated }\end{array}$ & 1 hour & Month 2,4,10 \\
\hline Team leader reflection sessions & $\begin{array}{l}6 \text { externally } \\
\text { facilitated }\end{array}$ & 1 hour & $\begin{array}{c}\text { Month } 1,3,6 \\
9,12\end{array}$ \\
\hline Individual Supervision & $\begin{array}{l}\text { Self-organised } \\
\text { by team }\end{array}$ & $\begin{array}{l}\text { Part of clinical } \\
\text { supervision }\end{array}$ & Ongoing \\
\hline
\end{tabular}




\begin{tabular}{|c|c|c|}
\hline & Interviews & Focus Groups \\
\hline & $n=28$ & $n=24$ \\
\hline Age (years) & 46 years, 8 months & 44 years, 2 months \\
\hline Time in Mental Health Services & 17 years, 9 months & 15 years, 9 months \\
\hline Time in post (months) & $62.9(59.6)$ & $54.4(40.1)$ \\
\hline \multicolumn{3}{|l|}{ Gender $n(\%) n(\%)$} \\
\hline $\begin{array}{l}\text { Male } \\
\text { Female }\end{array}$ & $\begin{array}{l}11(39) \\
17(61)\end{array}$ & $\begin{array}{c}7(29) \\
17(71)\end{array}$ \\
\hline \multicolumn{3}{|l|}{ Ethnicity } \\
\hline $\begin{array}{l}\text { White British } \\
\text { White Irish } \\
\text { White Other } \\
\text { Black/Black British-African/ } \\
\text { Black British-Caribbean/ Black } \\
\text { Other } \\
\text { Asian/Asian British-Other } \\
\text { Other } \\
\text { Missing }\end{array}$ & $\begin{array}{c}19(68) \\
2(7) \\
2(7) \\
1(4) \\
1(4) \\
1(4) \\
2(7)\end{array}$ & $\begin{array}{c}15(63) \\
1(4) \\
0 \\
6(25) \\
1(4) \\
0 \\
1(4)\end{array}$ \\
\hline \multicolumn{3}{|l|}{ NHS Trust } \\
\hline $\begin{array}{l}\text { South London and Maudsley } \\
\text { NHS Foundation Trust } \\
{ }^{2} \text { gether NHS Foundation Trust }\end{array}$ & $\begin{array}{l}19(68) \\
9(32)\end{array}$ & $\begin{array}{l}11(46) \\
13(54)\end{array}$ \\
\hline \multicolumn{3}{|l|}{ Job Role } \\
\hline $\begin{array}{l}\text { Staff } \\
\text { Team Leader }\end{array}$ & $\begin{array}{c}23(11) \\
3(89)\end{array}$ & $\begin{array}{l}18(25) \\
6(75)\end{array}$ \\
\hline \multicolumn{3}{|l|}{ Team } \\
\hline $\begin{array}{l}\text { Assertive Outreach } \\
\text { Support and Recovery } \\
\text { Forensic } \\
\text { Psychosis } \\
\text { Other }\end{array}$ & $\begin{aligned} & 0 \\
23 & (82) \\
2 & (7) \\
1 & (4) \\
2 & (7)\end{aligned}$ & $\begin{array}{c}4(17) \\
18(75) \\
0 \\
2(7) \\
0\end{array}$ \\
\hline \multicolumn{3}{|l|}{ Profession } \\
\hline $\begin{array}{l}\text { Nurse } \\
\text { Psychiatrist } \\
\text { Psychologist } \\
\text { Social Worker } \\
\text { Occupational Therapist } \\
\text { Support, Time and Recovery } \\
\text { Worker } \\
\text { Other (2 Associate Practitioner, } \\
\text { 1 Physio technician) }\end{array}$ & $\begin{array}{c}14(50) \\
4(14) \\
2(7) \\
2(7) \\
2(7) \\
3(14) \\
1(4)\end{array}$ & $\begin{array}{c}12(50) \\
2(8) \\
1(4) \\
4(17) \\
1(4) \\
2(4) \\
2(4)\end{array}$ \\
\hline
\end{tabular}

support their use of the intervention. The Personal Recovery training was co-facilitated by a professional trainer and a person with lived experience of mental illness, from the charity RETHINK mental illness. The Personal Recovery learning objectives of the training were: i) To understand the theory and aims of the three working practices, ii) To have role-play experience of introducing and using each working practice, iii) To understand that the working practices lead to action to support recovery - so collecting information is a means not an end in itself, iv) To understand that person-centered support is the goal, so individualized rather than invariant practice is the aim, and v) To be able to identify barriers and solutions around using the working practices in routine practice. The Coaching for Recovery training was delivered by a professional coach and trainer from SLaM partners, (a team of organizational consultants and coaches who offer consultancy and training). The learning objectives of the Coaching for Recovery training were: i) To demonstrate how a coaching approach supports the implementation of recovery focused practice, ii) To equip clinical and support staff with knowledge of the core competencies required for working effectively with a coaching style, iii) To develop the participant's capacity and enable them to embed a coaching style within their clinical practice, iv) To build the capacity and capability of teams, services and the organization to successfully implement the REFOCUS Intervention's three working practices through the development of a coaching ethos. Table 2 summarizes the implementation strategies used to support use of the intervention. For further information, Participant and Trainer manuals for the Coaching for Recovery training are free to download at www.researchintorecovery.com/refocus.

\section{Materials}

A semi-structured interview schedule was developed and modified following consultation with the REFOCUS Lived Experience Advisory Panel [23] and piloting (See Online Data supplement 1: Staff interview schedule). The focus group topic guide was designed to explore the team-level views and experiences of implementing recovery intervention(s) as set out in the REFOCUS manual, (see Online Data supplement 2: Focus group topic guide).

\section{Procedure}

Four pilot interviews were conducted six months into the intervention to identify early issues associated with implementing the intervention (CLB). The remainder of interviews and all the focus groups were conducted towards the end of the intervention period. The research team identified potential interviewees who met inclusion criteria using networking techniques, either having heard they were using the intervention from trainers, research colleagues, or their own contacts within intervention teams. They were then approached by a researcher who explained the purpose of the study. Participants were given an information sheet and an opportunity to ask questions prior to giving written consent. The majority of interviews and all focus groups took place at the participant's team base. Most of the individual, face to face staff interviews were conducted by one member of the research team (EC, ML, MJ, VB in SLaM, EP and KS at ${ }^{2}$ gether). All interviewers received interview training, regularly met and were closely supervised, for instance, in six interviews, a junior and senior researcher were present for training purposes. All interviews lasted between 10-100 minutes, though the majority were 60 minutes long. Recruitment continued until at least one interview had been conducted in each of the six intervention waves and category saturation was reached. Each focus group was led by two facilitators (ML \& GR in '2gether; ML \& EC in SLaM), who alternated leading the discussion and taking a co-facilitator role. Groups lasted between 60-100 minutes.

\section{Analysis}

Audio files of interviews and focus groups were professionally transcribed verbatim, then checked and anonymised by researchers. Transcripts were analyzed in NVivo 9 using Braun and Clarke's six phases for thematic 
analysis [24]. Analysis was concurrent with data collection allowing exploration of emergent themes in later interviews.

\section{Results}

The thematic analysis resulted in a main coding frame with four categories:

Intervention, Implementation strategies, Practice Change and Outcomes. The first category, Intervention, contained two sub-themes, Recovery-promoting relationships and Working practices, relating to the two of the four recovery practice domains identified in the conceptual framework of Recovery-oriented practice, namely "Supporting personally defined recovery", and "Working relationships" [14]. The Implementation strategies category includes the four main implementation strategies used to support community mental health teams and individual staff members implement the intervention (information sessions, recovery and coaching training and individual supervision). Three sub-themes were identified in the third category, Practice Change, relating to staff knowledge, attitudes and perspectives on changes in their relationships with service users, and finally the four category, Outcomes, contained two sub-themes, Empowerment of service users and staff, and Team based approach to Recovery. Table 4 provides a hierarchy of the main themes and subthemes.

\section{Theme 1. Intervention}

\section{Recovery-promoting relationships}

The Recovery-promoting relationships intervention component focused upon how clinicians and service users interact and worked ${ }^{2}$ gether. The development of a valuesbased, collaborative relationship was supported through the use of a coaching style of interacting and team-based recovery training and reflection sessions.

Coaching: The majority of staff were very receptive to the idea of incorporating the coaching skills and approach within their clinical practice and found the training prepared them for having tough as well as motivating, empowering conversations with service users. Staff found the concept of three styles demonstrated in training (mentoring, directing and coaching) a helpful way of thinking about interactions. It reminded them that they have alternative options which may be more or less appropriate and beneficial, for different situations, service users, and stages of therapy.

'I've never done that before [...] I found it was a lot gentler and for some service users that would have been really good, some of those approaches were less intrusive and I think probably everyone found that'. (Focus group 2, '2gether).

The core coaching competencies of exquisite listening, asking powerful questions, contracting using the language of the service user, and reflection were identified as especially valuable, refreshing micro communication skills as well as offering ways of dealing with difficult situations. Coaching also helped staff be more explicit in their interactions with service users.

II have been making a more conscious effort to, not just have that style in the way I talk, but to say to someone "I'm not sitting here thinking I know the answers" or "I think you'll find the answers as you go forward in your life to these issues that

\begin{tabular}{|c|c|}
\hline \multicolumn{2}{|c|}{ 1. Intervention } \\
\hline \multirow[t]{2}{*}{ 1.1 Recovery-promoting relationships } & 1.1.1 Coaching skills and approach \\
\hline & 1.1.2 Partnership project \\
\hline \multirow[t]{4}{*}{ 1.2 Working practices } & 1.2.1 Values and treatment preferences \\
\hline & 1.2.2 Strengths \\
\hline & 2.2.3 Personally- valued goals \\
\hline & 2.2.4 Electronic recording of working practice conversations \\
\hline \multicolumn{2}{|c|}{ 2. Implementation strategies } \\
\hline \multicolumn{2}{|l|}{2.1 Information sessions } \\
\hline \multicolumn{2}{|l|}{ 2.2 Personal recovery training and reflection sessions } \\
\hline \multicolumn{2}{|l|}{2.3 Coaching for Recovery training } \\
\hline \multicolumn{2}{|l|}{2.4 Individual Supervision } \\
\hline \multicolumn{2}{|c|}{ 3. Practice change } \\
\hline \multicolumn{2}{|l|}{ 3.1 Staff knowledge of Personal Recovery } \\
\hline \multirow[t]{3}{*}{ 3.1 Staff attitudes towards Personal Recovery } & 3.2.1 Recovery now seen as part of role \\
\hline & 3.2.2 Broader focus upon personal recovery \\
\hline & 3.2.3 Challenge to clinician's illusion \\
\hline \multirow[t]{2}{*}{ 3.3 Staff perspectives on relationships } & 3.3.1 Quality of relationship \\
\hline & 3.3.2 Power balance \\
\hline \multicolumn{2}{|c|}{ 4. Outcome } \\
\hline \multicolumn{2}{|l|}{ 4.1 Empowerment of staff and service users } \\
\hline \multirow[t]{3}{*}{ 4.2 Team based approach to recovery } & 4.2.1 Dedicated time for team building \\
\hline & 4.2.2 Developing culture of challenging practice \\
\hline & 4.2.3 Recovery practice problem-solving \\
\hline
\end{tabular}


we're talking about" [...] I'm being less implicit more explicit'. (Interview, ID242011, Psychologist, SLaM).

Coaching was described as being enjoyable, inspiring and beneficial. Some staff noticed that working as a coach contrasted with approaches they might adopt, though they did say that coaching shared similarities to motivational interviewing and solution focussed therapy.

'People are trying to think about how best to have a shared project with this person and there's going to be a journey they'll take ${ }^{2}$ gether, rather than one that's enforced on the other person. I think that's clearly the most positive thing, the life coach rather than the rather than the coercing professional'. (Interview, ID412032, Psychiatrist, SLaM).

Personal recovery training and reflection sessions: Participants reported being confused between the different types of sessions and generally there was a mixed response to them. Some staff described the personal recovery training as thought-provoking and used it to reflect upon their own personal and team recovery-oriented values and practice. Not everyone agreed that the less structured reflection sessions, were an effective use of time.

'I think the ones that felt less helpful were the ones where there were just an hour of team reflection, because it just felt like a generalised conversation really and I think a few of us felt that we didn't get a lot back from that'. (Interview, ID112003 Nurse, SLaM).

There were teams where the Personal Recovery training was met with considerable resistance and staff described some sessions as uncomfortable to participate in because they were full of conflict and tension, though for many this did improve with time. Others felt the training was unnecessary and offered nothing new, because they already practiced in a recovery-oriented way. These issues are deal with in greater depth elsewhere [22].

\section{Working practices}

The Working Practices intervention component focused upon what tasks and activities clinicians and service users do together.

Values and treatment preferences working practice: The Values and Treatment Preferences (VTP) interview guide was one of the resources provided in the REFOCUS manual. It gave staff the confidence and permission to initiate new conversations, particularly around sexuality and spirituality, which they felt had previously been off limits.

Staff who successfully used the VTP guide with their service users shared their success and became powerful advocates for the approach within their team.
'What really struck me was when she [staff member] said 'oh I tried out one of these [REFOCUS] tools and I found that absolutely fantastic (laugh)'. She was totally onboard with it and I would not have expected that and getting her to use that. I think that shows how good the intervention was, I mean, she's not someone who would just do it. She's an open person as well, but she had set her mind against it [using REFOCUS tools] but still in spite of that actually tried it out, and then was able to say "oh yes, that was actually really helpful"'. (Interview, ID122021, Psychiatrist, SLaM).

Though there were examples of staff using the VTP guide in a rigid, inflexible way and concluding it was a bit too long and complicated, or that not all questions were suitable for everyone.

Strengths assessment working practice: Adopting a strengths approach led staff to have unexpected conversations with service users, which deepened their understanding of service users' perspectives. Noticing and celebrating the positive aspects of a person's identity rather than their deficits and problems led to clinician's re-evaluating their views of individual service users and opening up new ways of relating and possibilities for the future.

'It's amazing how their strengths are completely different from what I think their strengths are. I have learnt so much from my client, it's like I have known you almost two years but I would never think that could come out of you. It brings out their potential, a part of them I am not really aware of, or haven't taken notice of, but that has made me understand him differently'. (Interview, ID122014, Nurse, SLaM).

Strengths were often identified by service users as personal characteristics and a drawback to the strengths assessment worksheet was their exclusion. Some staff felt at a loss when service users reported they had little or no strengths and appeared to have had insufficient training to deal with this scenario.

Goal-striving working practice: Participants talked about this working practice the least, frequently explaining that they have always done this. They reported that the coaching training had emphasized the need to continuously evaluate progress and use coaching skills such as contracting and providing feedback as part of the goal-setting process. One interviewee explained how she was surprised to learn a service user who she knew well, had a life goal of setting up her own nursery and was undertaking a childcare qualification to help her achieve this, which lead to her re-evaluating her long held view them:

'I underestimated that the response would be "yeah, I've got goals". What has made it quite interesting is that before I did this training I knew she was doing these [childcare] courses but I never saw it as, in terms of goal-setting'. (Interview, ID122011, Nurse, SLaM). 
Particular barriers to this working practice were that some staff wanted to protect service users, felt their goals were not realistic or were not sufficiently motivated to achieve their goals. Again, this suggests strategies for dealing with these issues and practical difficulties may have been insufficiently covered in the training.

\section{Theme 2: Practice changes}

There were staff practice changes at both an individual and a team level. At an individual, there were changes in staff knowledge and attitudes about personal recovery, and in their relationships with service users. At a team level, there were improved relationships within the team and space to focus on developing a team-based approach to recovery.

Individual practice changes

Staff knowledge of personal recovery: Some staff found the training and manual provided a structured approach to work in a recovery-oriented way, others thought it had not made any conspicuous impact on their practice. Staff who did notice changes gave examples of how the focus of their clinical work had expanded or shifted to being more personalized.

'Sometimes you might only focus on giving someone their medication, but when this [REFOCUS] was brought in, it broadened my knowledge, implementing what was being taught. You become complacent, you might think, "oh this person has been on my caseload for five years and they're not moving on', but then it gives you different aspects to you can focus on"'. (Interview, ID342014, Nurse, SLaM).

Staff attitudes towards personal recovery: There were examples of where clinicians had reflected upon the purpose of mental health services and importance of keeping collaborative working with service users on the agenda.

'It also surfaced I think aspects about people's assumptions about what is it that that we are doing as a core business and I think we were able to have a kind of conversation around that'. (Interview, ID332002, Psychiatrist, SLaM).

'The issue of power and control is something that's very overlooked in the system and I think collaboration, including helping clients themselves feel that they are in charge is something that isn't even on the radar ordinarily'. (Interview, ID412003, Psychologist, SLaM).

The intervention reminded some clinicians to broaden their focus beyond a medical model-driven style of questioning and revisit decisions where they may have been risk-averse and over-protective:

'I think it's just useful and it prompts you to ask questions. Sometimes you get fixed in a medical model and it's all about medication and side effects and stuff. It's good to remind yourself of other things that people value'. (Interview, ID122026, Nurse, SLaM).
'It's not that we don't allow people to have choices, but that we often assume that patients know they have a choice. ...sometimes I'm not so sure that they do know that they can take it or leave it'. (Interview, ID136, Team leader, SLaM).

It also led some staff to revise their belief that no one recovers from severe mental illness and to question their own assumptions about their service user motivations, capabilities, goals, aspirations, strengths and personal attributes.

'It's certainly made me think more and not take so much for granted, not to assume that I know it all about people who I've known for a long time, which is a bit of a challenge really'. (Interview, ID136, Team leader, SLaM).

'I just didn't see a difference between somebody who's had years of really being limited by schizophrenia and myself. I just saw a human being, just looking at life and saying "I'm gonna go as far I can, the sky's the limit". (Interview, ID122011, Nurse, SLaM).

Staff changes in relationship: Participants felt that using the intervention helped refresh long-term clinician-service user relationships and helped them develop their knowledge and understanding of service users' lives though noticing other, positive aspects of their individual identity.

'It's definitely strengthening the relationship. I think it's to do with perceiving an individual as a person'. (Interview, ID122011, Nurse, SLaM).

The intervention served as a reminder to staff that using a directive style of interaction is not always appropriate. It helped some staff strike the difficult balance in clinical practice between knowing when to take a step back and allow a service user to manage a task or situation alone and when to step in and support.

'It's made me really think, what is it I'm doing? Am I making myself more important in this person's life than I need to be or should be? In this role sometimes, you do get into an almost a preaching, telling role [Laughs] and sometimes it's nice to be reminded that we're there for their benefit. You want to take charge of your life, so we will withdraw'. (Interview, ID134, SLaM).

When asked more generally about the impact of the intervention, some staff credited the REFOCUS intervention with empowering them and/or their service users.

'Asking them what they think needs to happen instead of telling. I just stand there for a moment and just say 'What do you think needs to happen?' and that was really powerful I think'. (Interview, 122021, Psychiatrist, SLaM).

'My anxiety level has sort of reduced in terms of when I'm making decisions. Now I've got confidence to trust that with these techniques if the person is able to come up with their own solution it's better for them'. (Focus group 4, SLaM). 


\section{Team based approach to recovery}

Providing reflection sessions and recovery training to the whole clinical team was viewed very favorably. They reported it improved team relationships and dynamics by providing precious, dedicated time for everyone to be a team and created a safe place for sharing work pressures and challenging one another's practice.

'I feel I can challenge colleagues now and people won't take exception to being challenged. Team discussions give people more confidence that they've been doing the right thing, or be able to go back to clients and say "you know what, you were right and last time, I was wrong"'. (Focus group 3, SLaM).

It also provided a space for problem-solving how to overcome obstacles to recovery practice and developing team plans for taking the recovery agenda forward. Clinicians were very aware that they were not working in a vacuum, and needed to liase with other agencies and inpatient services with a different agenda. They reported the intervention had enabled them to have the time and space to identify many obstacles that needed to be overcome when working within ethical and legal frameworks, statutory requirements and within a complex organizational system. In particular, they recognized the conflict that can be present between an individual's goals, organizational goals and statutory responsibilities and the need to discuss strategies for marrying these different expectations and requirements.

Fundamentally, it provided the reportedly rare opportunity for teams to develop a shared vision of recovery and decide a plan of action.

'It's working as a team, using different skills and identifying the recovery needs right from the outset, which I'm not sure that we always did'. (Focus group 2, '2gether).

'Having the time to reflect upon our culture, contributions we make individually to the team, where we want to go and having some sort of shared vision about where we want to go, being on board to go there together - that is most valuable thing, that space to think about how we want to move forward'. (Focus group 3, SLaM).

\section{Discussion}

The study aimed to investigate community mental health staff experiences of using the REFOCUS intervention. Staff were especially positive about the coaching training and the resources within two of the three working practices, highlighting the Values and Treatment preferences guide and the Strengths Assessment Worksheet, as giving them permission for new, structured conversations which led to finding out more about service users. The intervention also facilitated the development of recovery practice by supporting the development of team culture, structures and processes. The REFOCUS training was provided at to the whole community mental health team at the same time, which meant it was not possible to get everyone together for each training session, but arrangements were put in place for staff to share their learning with absent colleagues and trainers ensured that staff felt able to prioritise clinical demands, if needed.

The quantitative findings from the REFOCUS trial showed that although patient rated personal recovery scores (i.e. the Questionnaire of Processes of Recovery - the trial's primary outcome measure) did not differ between the control and intervention groups, implementation of the intervention varied. In teams with high participation scores (adherence to the intervention) there were higher staff-rated scores for recovery-promotion behaviour change and patient-rated Personal Recovery interpersonal scores. There was a trend for patients treated in the REFOCUS group to incur $£ 1,062$ lower service use costs than those in the control group [33]. Clinical and personal recovery outcomes were found to be independent, confirming that personal recovery is a distinct construct [34]. Patients reported that the intervention supported the development of open and collaborative relationships with staff and new conversations about values, strengths and goals [35]. Organisational readiness and training effectiveness were important factors influencing implementation [22]. The REFOCUS researchers have since worked with colleagues in Australia to replicate the trial in primary and secondary care settings [36,37].

\section{Strengths and limitations}

The number of research studies which look specifically at supporting recovery practice from a staff perspective is limited. Existing qualitative studies tend to examine experiences and views relating to organisational level implementation of recovery initiatives $[25,26]$ focus upon an the application of recovery practice principles where there may be specific tensions, such as risk [27] or involuntary treatment [28], or focus upon recovery practice within a particular treatment model [29] or setting [30]. This study examines staff perspectives on a complex, team-level intervention to support recovery and gives an insight into their views around individual components of such an intervention. The study sample of 49 participants is relatively large for a qualitative study. The characteristics of participants are diverse and include staff from a range of professional backgrounds, with differing levels of experience and seniority and who work within specialist and generic types of community based mental health team.

Some caution should be taken when considering the findings. The sample inclusion criteria specifically stated that staff should report their use of at least some parts of the intervention, so we do not claim the sample represents the entire population of staff working in intervention teams. Although we did explore the barriers and enablers to implementation with this sample, reported elsewhere, [22] staff who admitted they did not implement the intervention 
were excluded from this study because they would not have been able to discuss their experiences of using the intervention, which was the specific aim of this study. Finally, the research programme did not include a long-term follow up to investigate the sustainability of the REFOCUS intervention, so it is not known whether practice changes were continued, on beyond the completion of the research study.

\section{Implications for clinical practice}

There are three key implications for clinical practice.

Use of Coaching as a means of developing recoverypromoting relationships: On the basis of these findings, we would support others who have recommended coaching as a means of developing recovery promoting relationships [31]. Staff valued the concrete guidance and skills training they were given to prepared them for having tough as well as motivating, empowering conversations with service users. This has wider clinical relevance given that initiating tough conversations will always part of mental health practice and can be stressful for both clinicians and service users. Observing, rehearsing and reflecting on the clinical applications of different interaction styles of directing, mentoring and coaching helped clinicians decide how to strike a balance between when to provide help and when to step back. This clearly is fundamental to developing empowering relationships which facilitate recovery and has relevance for training of all mental health professionals.

Use of working practices: There is some evidence that using the three working practices helped clinicians expand the focus of care. Some of these conversations directly challenged clinician's views and assumptions about what their service users were capable of and thereby subtly changed and expanded their view of the person. Shifting staff attitudes and beliefs about the possibility of recovery from severe mental illness, their views of service user strengths, personal resources and capabilities is important step in changing towards a more recovery focused practice. In a qualitative study of 28 case managers in Indiana, USA, Sullivan and Floyd looked at differences in their recovery practice and beliefs about the likelihood of service user recovery from mental illness [32]. They found that the most hopeful case managers, who had often witnessed individuals succeeding in unanticipated ways, focused on outcomes which went beyond symptom relief and compliance with medication.

Team-based approach to recovery practice: The teambased approach to training and reflection sessions was singled out as being important in enabling teams to support and critically challenge one another, develop a team recovery identity and a team culture which was conducive to supporting and sustaining recovery practice.

\section{Acknowledgements}

We would like to thank the staff and team leaders that took part in the main trial and volunteering to share their thoughts and experiences by participating in these interviews and focus groups. We would also like to express our gratitude to our trainers for their patience and flexibility and those that assisted them in provided administrative support scheduling the training sessions with clinical teams or offering professional supervision and support.

\section{Funding}

This article presents independent research funded by the NIHR under its Programme Grants for Applied Research Programme (Grant Reference Number RP-PG-0707-10040), and in relation to the National Institute for Health Research (NIHR) Biomedical Research Centre for Mental Health at South London and Maudsley NHS Foundation Trust and Institute of Psychiatry, King's College London. The views expressed in this publication are those of the authors and not necessarily those of the NHS, the NIHR or the Department of Health.

\section{Ethical approval}

The study was approved by East London Research Ethics Committee, (Ref. 11/LO/0083) on 22/2/11.

\section{Trial registration}

Current Controlled Trials ISRCTN02507940

\section{References}

1. Bonney S, Stickley T. Recovery and mental health: A Review of the British Literature. J Psychiatr Ment Health Nurs. 2005; 15: 140-153. PubMed: https://www.ncbi.nlm.nih.gov/pubmed/18211561

2. Everett B, Adams B, Johnson J, Kurzawara G, Quigley M, et al Recovery rediscovered: Implications for the Ontario mentla health system. In: Association CMH, editor.: Ontario Division; 2003.

3. Australian Health Ministers' Advisory Council. A national framework for recovery-oriented mental health services: Policy and Theory. In: ageing DoHa, editor.: Commonwealth of Australia; 2013.

4. Anthony W. Recovery from Mental IIness: The Guiding Vision of the Mental Health Service System in the 1990s. Psych Rehab J. 1993;16: 11-23.

5. Farkas M, Gagne C, Anthony W, Chamberlin J. Implementing Recovery oriented evidence based programs: Identifying the Critical Dimensions. Community Ment Health J. 2005; 41: 141 - 158.

PubMed: https://www.ncbi.nlm.nih.gov/pubmed/15974495

6. Davidson L, Rowe M, Tondora J. A Practical Guide to RecoveryOriented Practice: Tools for Transforming Mental Health Care USA: Oxford University Press; 2008.

7. Le Boutillier C, Slade M, Lawrence V, Bird V, Chandler R, et al Competing Priorities: staff perspectives on supporting recovery. Administration and Policy in Mental Health and Mental Health Services Research. in press.

8. Slade M, Amering M, Farkas M, Hamilton B, O’Hagan M, et al. Uses and abuses of recovery: implementing recovery-oriented practices in mental health systems. World Psychiatry. 2014; 13: 12-20. PubMed: https://www.ncbi.nlm.nih.gov/pubmed/24497237

9. Gilburt H, Slade M, Bird V, Oduola S, T. C. Promoting recovery- 
oriented practice in mental health services: a quasi-experimental mixed-methods study. BMC Psychiatry. 2013; 13: 167.

PubMed: https://www.ncbi.nlm.nih.gov/pubmed/23764121

10. Repper J, Perkins R. Social Inclusion and Recovery: An Approach for Mental Health Practice: Balliere-Tindall; 2003.

11. Rapp C, Goschka R. The Strengths model: Case Management for People with Psychiatric Disabilities. 2nd ed. Oxford: Oxford University Press; 2006.

12. Slade M, Bird V, Le Boutillier C, Williams J, McCrone $P$, et al. The REFOCUS Trial: A cluster randomised controlled trial of a pro-recovery intervention within community based mental health teams. 2011; 11: 185. PubMed: https://www.ncbi.nlm.nih.gov/pubmed/22112008

13. Bird V, Leamy M, Le Boutillier C, Williams J, Slade M. REFOCUS: promoting recovery in community mental health services. 2011.

14. Le Boutillier C, Leamy M, Bird V, Davidson L, Williams J, et al. What does recovery mean in practice? A qualitative analysis of international recovery-oriented practice guidance. Psychiatr Serv. 2011; 62: 1470-1476. PubMed: https://www.ncbi.nlm.nih.gov/pubmed/22193795

15. Leamy M, Bird V, Le Boutillier C, Williams J, Slade M. Conceptua framework for personal recovery in mental health: systematic review and narrative synthesis. Br J Psychiatry. 2011; 199: 445-452.

PubMed: https://www.ncbi.nlm.nih.gov/pubmed/22130746

16. Bird V, Leamy M, Tew J, Le Boutillier C, Williams J, et al. Fit for purpose? Validation of the conceptual framework of personal recovery with current mental health service users. Aust N Z J Psychiatry. in press

17. Slade $M$, Leamy $M$, Bacon $F$, Janosik $M$, Le Boutillier $C$, et al. International differences in understanding recovery: systematic review. Epidemiol Psychiatr Sci. 2012; 21: 353-364.

PubMed: https://www.ncbi.nlm.nih.gov/pubmed/22794507

18. Bird V, Le Boutillier C, Leamy M, Larsen J, Oades L, et al. Assessing the strengths of mental health service users - systematic review. Psychol Assess. 2012; 24: 1024-1033.

PubMed: https://www.ncbi.nlm.nih.gov/pubmed/22708573

19. Shanks V, Williams J, Leamy M, Bird V, Le Boutillier C, et al. Measures of personal recovery: systematic review. Psychiatr Serv. 2013; 64 974-980. PubMed: https://www.ncbi.nlm.nih.gov/pubmed/23820592

20. Williams J, Leamy M, Bird V, Harding C, Larsen J, et al. Measures of the recovery orientation of mental health services: systematic review. Soc Psychiatry Psychiatr Epidemiol. 2012; 47: 1827-1835. PubMed: https://www.ncbi.nlm.nih.gov/pubmed/22322983

21. Grant A, Treweek S, Dreischulte T, Foy R, Guthrie B. Process evaluations for cluster-randomised trials of complex interventions: a proposed framework for design and reporting. Trials. 2013; 14: 15 . PubMed: https://www.ncbi.nlm.nih.gov/pubmed/23311722

22. Leamy M, Clarke E, Le Boutillier C, Bird V, Janosik M, et al. Implementing a manualised intervention to support personal recovery: a qualitative study nested within a Cluster Randomised Controlled Trial. PLoS One. 2014; 9: e97091.

PubMed: https://www.ncbi.nlm.nih.gov/pubmed/24875748

23. Slade M, Bird V, Chandler R, Fox J, Larsen J, et al. The contribution of advisory committees and public involvement to large studies: case study. BMC Health Serv Res. 2010; 10: 323.

PubMed: https://www.ncbi.nlm.nih.gov/pubmed/21126353
24. Braun V, Clarke V. Using thematic analysis in psychology. Qualitative Res Psycho. 2006; 3: 34.

25. Hungerford $C$, Kench $P$. The perceptions of health professionals of the implementation of Recovery-oriented health services: A case study analysis. J Mental Health Training, Education and Practice. 2013; 8: $208-218$.

26. Piat M, Lai S. Service Providers' Experiences and Perspectives on Recovery- Oriented Mental Health System Reform. Psychiatr Rehabil J. 2012; 35: 289-296.

PubMed: https://www.ncbi.nlm.nih.gov/pubmed/22491368

27. Tickle A, Brown D, Hayward M. Can we risk recovery? Agrounded theory of clinical psychologists' perceptions of risk and recoveryoriented mental health services. Psychol Psychother.. 2014; 87: 96-110. PubMed: https://www.ncbi.nlm.nih.gov/pubmed/24497399

28. Courtney M, Moulding N. Beyond Balancing Competing Needs: Embedding Involuntary Treatment Within a Recovery Approach to Mental Health Social Work. Aus Soc Work. 2013.

29. Felton BJ, Barr A, Clark G, Tsemberis SJ. ACT Team Members' Responses to Training in Recovery-Oriented Practices. Psychiatr Rehabil J. 2006; 30: 112-119.

PubMed: https://www.ncbi.nlm.nih.gov/pubmed/17076054

30. Cleary M, Horsfall J, O'Hara-Aarons M, Hunt GE. Mental health nurses' views of recovery within an acute setting. Int J Ment Health Nurs. 2013; 22: 205-212.

PubMed: https://www.ncbi.nlm.nih.gov/pubmed/22882297

31. Bora R, Leaning S, Moores A, Roberts G. Life coaching for mental health recovery: the emerging practice of recovery coaching. Advances in Psychiatric Treatment. 2010; 16: 459-467.

32. Sullivan P, Floyd D. Spirit Lifting: Hope and Recovery in Case Management Practice. Families in Society: J Contemporary Social Serv. 2013; 94: 38.

33. Slade M, Bird V, Clarke E, Le Boutillier C, McCrone P, et al. Supporting recovery in patients with psychosis using adult mental health teams (REFOCUS): a multi-site cluster randomised controlled trial. Lancet Psychiatry. 2015; 2: 503-514.

PubMed: https://www.ncbi.nlm.nih.gov/pubmed/26360446

34. Macpherson R, Pesola F, Leamy M, Bird V, Le Boutillier C, et al. The relationship between clinical and recovery dimensions of outcome in mental health. Schizophr Res. 2016 175, 1-3, 142-147. PubMed: https://www.ncbi.nlm.nih.gov/pubmed/26527245

35. Wallace G, Bird V, Leamy M, Bacon F, Janosik M, et al. Service user experience of the REFOCUS intervention. Soc Psychiatry Psychiatr Epidemiol. 2016; 51: 1275-1284.

PubMed: https://www.ncbi.nlm.nih.gov/pubmed/27365099

36. Meadows G, Brophy L, Shawyer F, Enticott J, Fossey E, et al REFOCUS-PULSAR recovery-oriented practice training in specialist mental health care: a stepped-wedge cluster randomised controlled trial. Lancet Psychiatry. 2019; 6: 103-114.

37. Shawyer F, Enticott J, Brophy L, Bruxner A, Fossey E, et al. The PULSAR Specialist Care protocol: a stepped-wedge cluster randomized control trial a training intervention for community mental health teams in recovery-oriented practice. BMC Psychiatry. 2017; 17: 172. PubMed: https://www.ncbi.nlm.nih.gov/pubmed/28482829 